\title{
Ocular Prosthesis: Face to New Look
}

\section{Janak Poudel ${ }^{1,2 *}$, Archana Murthy ${ }^{3}$ and Premnath Krishnasamy ${ }^{4}$}

${ }^{1}$ B. Optom, Vittala International Institute of Ophthalmology, Bangalore, India

${ }^{2}$ Consultant Optometrist, Megha Hospital, Kathmandu , Nepal

${ }^{3}$ Consultant Ocular Prosthetic Department, Prabha Eye Clinic and Research Centre,

Vittala Eye Hospital, Bangalore, India

${ }^{4}$ Assistant Professor, Vittala International Institute of Ophthalmology, Bangalore, India

*Corresponding Author: Janak Poudel, B. Optom, Vittala International Institute of

Ophthalmology, Bangalore, India and Consultant Optometrist, Megha Hospital,

Kathmandu , Nepal
Received: June 13, 2021

Published: July 19, 2021

(C) All rights are reserved by Janak Poudel., et al.

\begin{abstract}
Our eyes are the windows to our soul as well as the first features of the face to be noted. The human facial disfigurement related with loss of an eye can lead towards extensive levels of physical and emotional problems. The rehabilitation of an affected patient who has suffered the psychological trauma of an ocular loss requires a prosthesis which provides the most efficient cosmetic and useful end result. An ocular prosthesis is a simulation of human anatomy that uses the prosthetic materials to create an illusion of a normal healthy eye and surrounding tissue as well as to preserve the volume of eye socket. A custom made ocular prosthesis has many advantages as compared to stock shell ocular prosthesis.
\end{abstract}

Keywords: Ocular Prosthesis; Custom Ocular Prosthesis; Stock Shell Ocular Prosthesis; Polymethyl Methacrylate

\section{Introduction}

Our eyes are the windows to our soul as well as the first features of the face to be noted [1]. We modify and alter our conduct and act according to our interpretations whether that's related to our physical surrounding, social interactions or emotional contact. The loss of an eye is mostly due to congenital defect, irreparable trauma, painful blind eye, various eye diseases and sympathetic ophthalmia. The human facial disfigurement related with loss of an eye can lead towards extensive levels of physical and emotional problems. The rehabilitation of an affected patient who has suffered the psychological trauma of an ocular loss requires a prosthesis which provides the most efficient cosmetic and useful end result.

An ocular prosthesis is a simulation of human anatomy that uses the prosthetic materials to create an illusion of a normal healthy eye and surrounding tissue as well as to preserve the volume of eye socket. An ocular prosthesis may be available readymade shells or can be custom made. A stock ocular prosthesis can be given in short period of time and cost factor is taken into consideration. But a stock ocular prosthesis has many disadvantages, such as illfitting, improper shade matching with natural adjacent eye, can give proptosis effect, whereas a custom made ocular prosthesis have proper adaptation, it may have slight movements and matches the iris position as that of the adjacent natural eye. The surgical procedures in the removal of an eye are classified into: a) Evisceration, b) Enucleation, c) Exenteration [2,3].

An ideal socket for the fitting of an ocular prosthesis should have:

- A well placed implant with the extraocular muscle attached. 
- Adequate superior and inferior for positive retention of the prosthesis.

- A palpebral fissure equal in size and shape to the tissues of the natural eye.

- Adequate anterior posterior depth to the socket.

- $\quad$ Adequate support of the superior and inferior tarsal plates.

- Minimum scar tissue adhesions in the socket.

- $\quad$ Adequate mobility of the eyelids $[1,4]$.

\section{Clinical considerations}

- A patient's history including the details of the nature of the disease, its mode of onset with reference to the visual status and recurrence should be taken. Family history along with congenital or hereditary anomalies such as cataract, albinism and iris deformities, etc.

- Examination of the external adnexa and socket should be done.

- Evaluate presence and extent of any superior sulcus defect, ptosis or horizontal eyelid laxity.

- The socket is examined to determine the presence of an implant and the degree of mobility.

- Any conjunctival discharge?

- If the patient has previously worn any prosthesis, the type, tolerance, and difficulties, if any, experienced are also noted.

- A growing child will require periodic enlargement of the prosthesis gradually over a period of years to aid in the development of the lids and other soft tissues lining orbital bone margins [4-10].

\section{Chemical used}

The chemicals used in making ocular prosthesis are polymethyl Methacrylate monomer and polymer. Polymethyl Methacrylate (PMMA) it is colourless transparent plastic with an excellent outdoor life period and good strength. Optical clarity is the main feature of this plastic [11].

\section{Methods}

Taking an impression

First explain the procedure to patient and make them feel comfortable.

Then insert the impression tray in cul-de-sac, and ask patient to look straight. Take $10 \mathrm{cc}$ of water in bowl, put measured alginate powder in it and mix it to a flowing paste. Cooler the temperature of water longer will be the setting time. Now load this paste into syringe and make sure there is no any air gap in the paste. Inject adequate alginate in the tray which is ready in the patient's eye so that it will fill up the entire sac and starts flowing out of the eye penetrating through the holes of the tray.

Once the alginate is set, remove the impression gently from the eye and cutoff the extra part.

Take a glass, add some measured alginate powder and water and mix it well to make a paste. Put and hold the impression in this paste and allow it to set.

Once it is set take out the content from the glass and cut it into half so as to remove the impression tray from it. Put the content back into the glass and heat the white carving wax and pour it into the hole so as to get the wax mould. Open the two halves of the alginate and take out the set wax in it, this is your wax model [9-11].

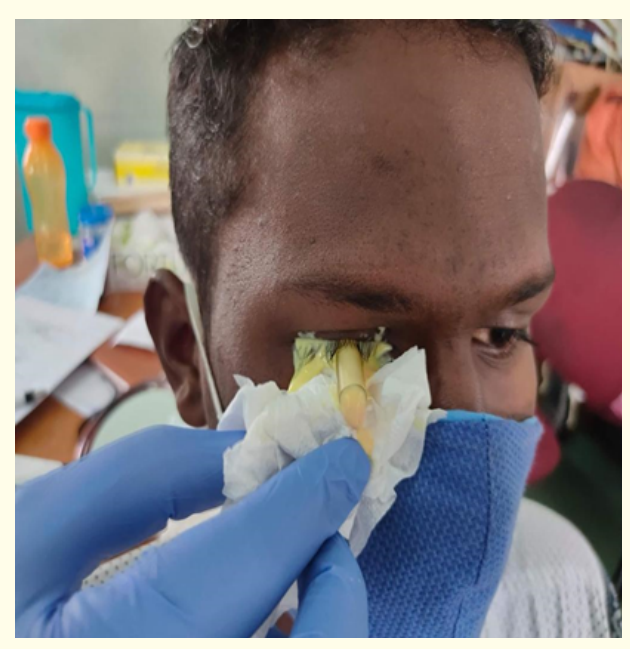

Figure 1: Taking an impression. 


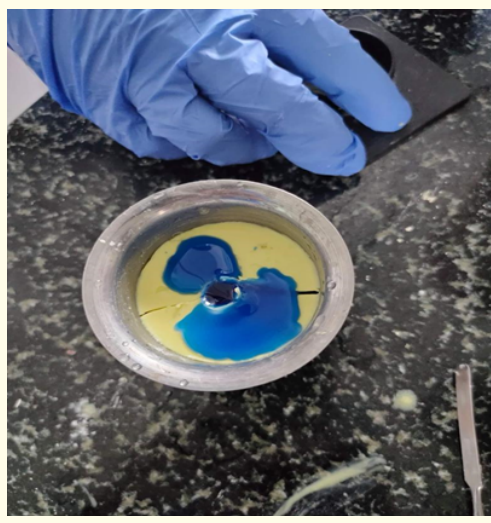

Figure 2: Wax model.

\section{Sculpting the wax}

Take an aluminum iris button of exact size as natural eye and place it in exact position where iris should be in your wax model. We can measure the interpupillary distance from the nose to good eye and try to keep the same in artificial eye to match the exact position of iris. Carving with the scalpel, using hot flame and tissue, we can smooth the wax eye. To remove out the irregularities on the back surface and make it smooth you can use cotton bud.

To match the other's eye palpebral fissure, protrusion, total closure of eyelids, superior sulcus, lower lid crease, shape of the lids, we can add or subtract the wax and sculpt the wax model. As soon as your aluminum button is set at proper location and in proper plane along with the sculpting of wax model, then proceed with making a plaster mould of it [11,15-17].

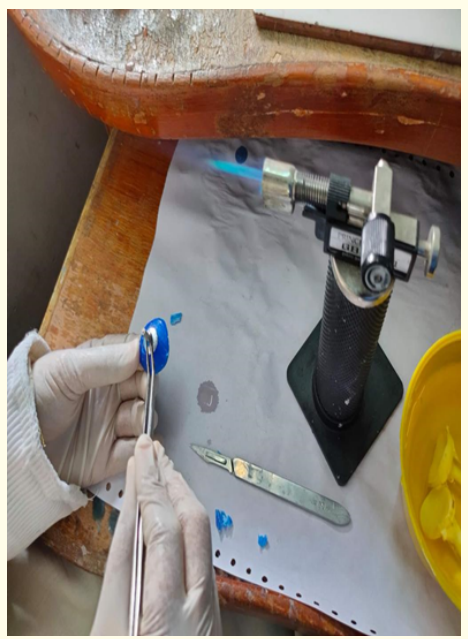

Figure 3: Attaching aluminum iris button.

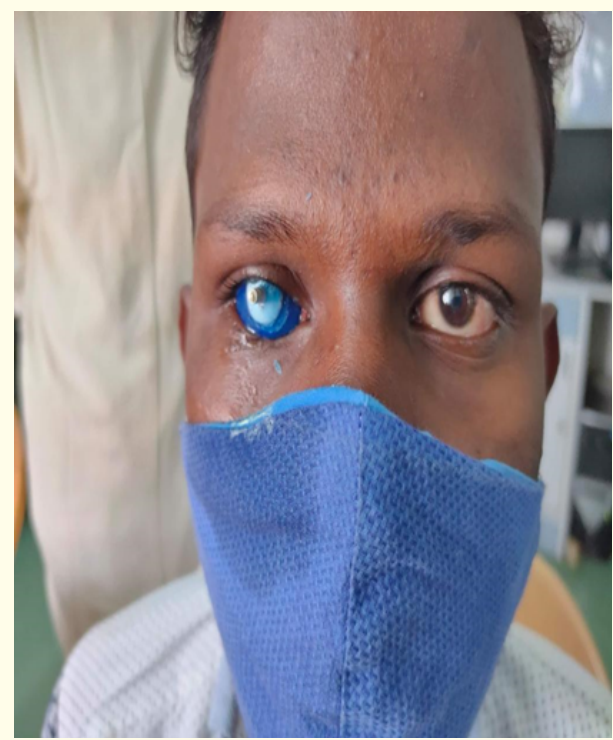

Figure 4: Iris orientation on scleral wax pattern.

\section{Making a mould in plaster}

Three different types of plaster can be used, they are:

- White plaster, faster in setting, but weaker in strength.

- Yellow or green plaster which is relatively tough and sets faster than blue plaster, but slower than white plaster.

- Blue plaster or dental stone which is toughest among all and takes longer time to set.

Mix the plaster in water, the quantity of plaster need to be just sufficient to soak up all of the water in bowl. Now put this plaster in the eye flask just to fill it completely till top and allow it to settle. Put the wax model with aluminum button attached to it in this flask button stem going deep down and the edge of the mould just touching the plaster from all the sides possibly equally. To remove the button and plaster at end apply Vaseline to the flask and aluminum button stem.

Again mix the plaster and ensure that there are no air bubbles and fill the other half of the flask with it and put some plaster on the back of the wax mould also. Put the two half of the flask together and press it firmly, whatever extra plaster is present will come out of it, allow the plaster to set now. Once the plaster is set properly you can separate the two half of the eye flask and scrap out the wax and remove the aluminum button carefully. Now the plaster mould is ready for making the plastic cast out of it [11]. 


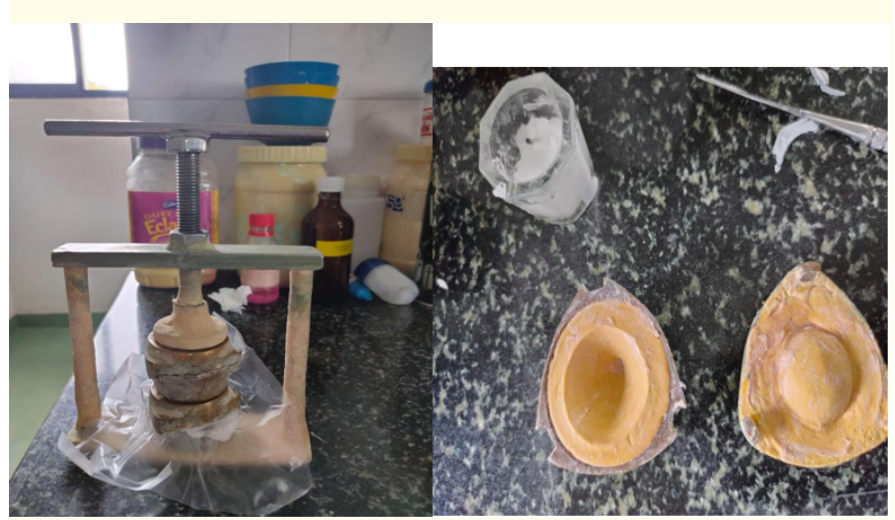

Figure 5: Plaster mould.

\section{Painting}

Painting the artificial eye we can use oil colors, crayon colors, plastic colors or all in combination using moly-poly syrup as medium. Using dry pigments they do last long, but if we use coloring pencils it gets lighter as the years pass. Fine red embroidery threads are placed on the scleral painting to mimic the blood vessels of the patient's natural eye, as well as it will give the three dimensional effect. Then the scleral part is layered with monomer polymer syrup to keep the blood-vessel fibers in place and allowed it to set. To give iris a natural look, it is better to do multilayer painting. i.e. paint more layers in many plains than one flat layer [11-17].

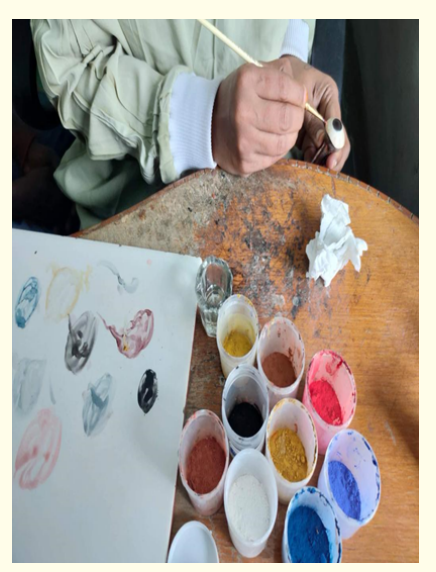

Figure 6: Painting.

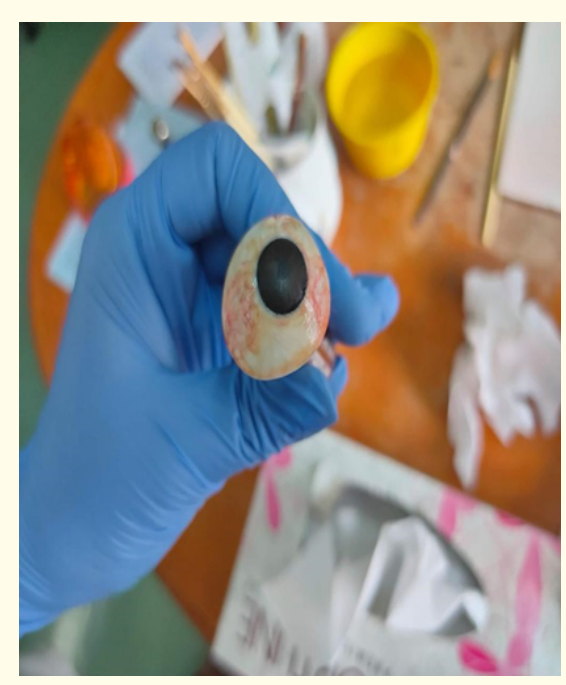

Figure 7: After putting red embroidery threads.

\section{Packing and flasking}

Packing and flasking is the chief step as it includes the characterisation of the prosthesis before packing with tooth coloured warmth cured polymethylmethacrylate of appropriate coloration, matching with the colour of the sclera of normal eye of the patient. A thin layer of heat cured clear PMMA was spread evenly in and around the iris. The characterisation is done so as to achieve the vitality necessary to give it a life like appearance and blend with the patient's natural appearance and cosmetics. After the characterisation, the mold was packed with heat cured tooth coloured PMMA of appropriate shade and kept for bench curing to enable complete polymerization and prevention of any excess unreacted monomer. This enables the minimization of porosities and gives a good finish to the prosthesis.

The eye socket is very sensitive to any surface roughness and irregularities so to prevent any residual monomer in prosthesis we use a lengthy curing cycle of 4 - 6 hours [17].

Final finishing and polishing of the prosthesis

Finished prosthesis requires a highly polished surface which would have a glass like finish to provide maximum adaptation and overall success of the prosthesis [17-19]. 


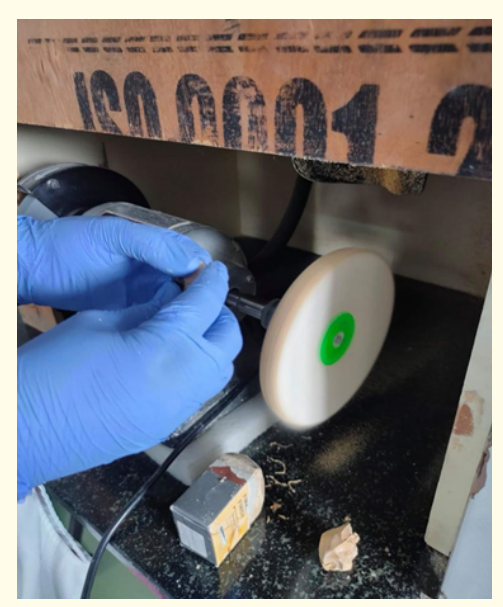

Figure 8: Polishing.

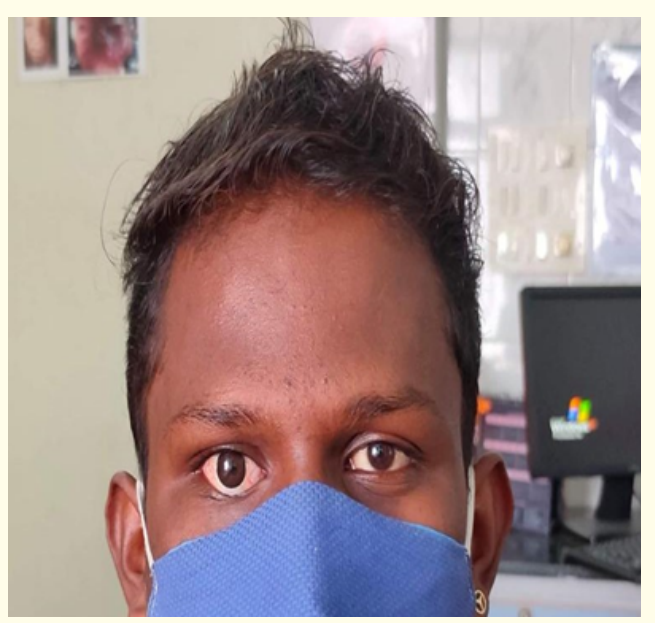

Figure 9: Final look.

\section{Dos and don't in prosthetic care}

\section{Minimal handling of the prosthesis:}

- The prosthesis can be removed once in a month and washed properly with cleaning soap and water. Always sleep with prosthesis in place.

- A plunger is provided to every patient which helps in easy removal and fitting of prosthesis.
- Frequent removal of the shell on a daily basis, may lead to discharge, watering and eyelid laxity, eyelid eversion and in adequately of the space to fit the shell.

Use of lubricating eye drops over the shell:

- Provides smooth surface for the eyelid movements over the shell.

- Washes off the debris deposited on the shell surface.

Yearly polishing of the prosthesis:

- Polishing helps to smoothen the regular surface and rounded edges which prevent friction between eyelids and prosthesis.

- This prevents any inflammatory reaction such as socket granuloma formation and giant papillary reaction in the eyelids.

A full framed protective polycarbonate glass should be worn to protect the normal eye from injury as well as camouflage the minimal differences between the normal eye and prosthetic eye.

Tips for fitting prosthesis on pediatrics

It has been divided into three approaches.

\section{First approach}

The replacement of the lost eye with a custom made ocular prosthesis should be carried out as early in life as possible. In case of toddlers with congenital deformities the remedy need to be carried out in the first 4 weeks of birth by placing a small ocular prosthesis (conformer) in the conjunctival socket. A conformer of a bigger size should be modified to prevent the cul-de-sac from shrinking kid grows [18].

\section{Second approach}

In most cases, a decent impression of the socket can be accomplished without the use of any medical aid, such as general anesthesia. But if the child is uncooperative, then an ocularist must take an impression of the socket under anesthesia.

Explain the child at their own understanding way or give them toy or chocolate so that they will get rid of fears. Important points to mention include the following: the impression does not hurt, it will be over quickly, it feels a bit cold [18-21]. 


\section{Third approach}

The third step is the prosthetic replication technique for the pediatric patient, including replication of the iris.

Iris painting

In newborn babies and toddlers as much as age of 12 to 15 months, we can see the iris surface is convex as well as the anterior chamber may be very shallow. Given our understanding of the anatomy of the eye, we know we need to paint on a bi-convex iris button in order to duplicate the iris and achieve the best visual result. With this particular shape, we are able to paint the foremost plane, providing the visual effect of a shallow anterior chamber. Anterior chamber depth keeps on increasing as well the iris gets flattens as the child grows. To provide the right depth effect on the iris of the prosthesis, now paint on a plano-convex iris button. To give the better visual effect of a deeper anterior chamber, the shape of iris button is used to paint the iris more in the background [19].

Sclera

According to the age of the child, change the color of the sclera.

Pupil size

Children older than 12 months generally have more dilated pupils; so it is better to make prosthesis having pupil diameter of 3 to $5 \mathrm{~mm}$. Maintaining the accurate pupil size in prosthesis is a challenging process because of the light, and even more if the child's iris is light in color. Widening and darkening the collarette of prosthesis makes an illusion to create the effect of changing pupil size. As a result, in brighter light, the pupil will appear small, while in dark light the deeper and wider collarette will make the pupil seem larger [19].

Iris position

The position of the child's iris should be slightly upward.

\section{Follow up}

Patient should be asked to follow up on day 1, 2 and 7 for follow up after prosthetic insertion. There after a 6 month to one year follow up was done for prosthesis evaluation and adjustment.

The integrity of the materials of a prosthetic will last for at least 10 years. However, most of the people will need a replacement at approximately 3 - 5 years due to the settlement of soft tissue in the eye socket. Need for replacement of prosthesis will also depend on the future settlement of the residual blind eye. Change of prosthesis once in every five years is ideal depending on changes taking place in socket.

\section{Discussion}

Effectively well shaped ocular prosthesis maintains its orientation once patient performs various movements [24]. Custom ocular prosthesis with PMMA can be made with exact fit and art although the prosthetic rehabilitation may be enhanced with the use of implants, can coordinate the movements with natural eye, as they are not always possible or feasible [26]. The processing of an ocular prosthesis for pediatrics as well as geriatrics is similar as an adult but child with an increasing age needs periodic enlargement of the prosthesis in order to accompany the expansion of the anophthalmic cavity. Although the stock ocular prosthesis can be given in a very short period of time, but a custom made ocular prosthesis provide better results as artistic as well as functionally.

Advantages of a custom ocular prosthesis are:

- $\quad$ Retains the shape of the socket.

- $\quad$ Prevents collapse of the lids.

- $\quad$ Provides proper muscular activity of the lids.

- Prevents accumulation of fluid in the cavity.

- Maintains palpebral opening similar to natural eye.

- Has a gaze similar to natural eye.

- Mimics coloration and proportions of natural eye.

- Conversational gaze can be achieved by maintaining precise interpupillary distance.

\section{Conclusion}

The custom made ocular prosthesis has been boon to those patient of facial disfigurement resulting from loss of an eye. Constructing the ocular prosthesis for pediatric patients are very challenging as well as time consuming process Treating such patients, an ocularist needs a good knowledge of the regional anatomy, artistic, very innovative, as well as an understanding of patient psychology and sensitivity. The custom ocular prosthesis are very cheap, affordable by patients, can be carried out in small clinical set up as well as it has provided good results from patient esthetics, acceptance and satisfaction points-of-view. 


\section{Bibliography}

1. Arbaz Sajjad. "Ocular Prosthesis - A simulation of Human Anatomy: A Literature Review”. Cureus (2012).

2. Perman KI and Baylis HI. "Evisceration, enucleation and exenteration". Otolaryngologic Clinics of North America 21 (1988): 171-182.

3. Lubkin V and Sloan S. "Enucleation and psychic trauma". Advances in Ophthalmic Plastic and Reconstructive Surgery 8 (1990): 259-262.

4. Parr GR., et al. "Surgical considerations in the prosthetic treatment of ocular and orbital defects". Journal of Prosthetic Dentistry 49 (1983): 379-385.

5. Doshi P and Aruna B. "Prosthetic management of patient with ocular defect". Journal of Indian Prosthodontic Society 5 (2005): 37-38.

6. Ocular Prosthesis. (2011).

7. Sykes LM. "Custom made ocular prosthesis: A clinical report". Journal of Prosthetic Dentistry 75 (1996): 1-4.

8. Cain JR. "Custom made ocular prosthesis". Journal of Prosthetic Dentistry 48 (1982): 690-694.

9. Welden RB and Niiranen JV. "Ocular prosthesis". Journal of Prosthetic Dentistry 6 (1956): 272-278.

10. Barlett S and Moore D. "Ocular Prosthesis: A physiologic system”. Journal of Prosthetic Dentistry 29 (1973): 450-459.

11. Chandrasekhar Chawan. "Ocular Prosthesis". 83-165.

12. Allen L and Webster H. "Modified impression method of artificial eye fitting". American Journal of Ophthalmology 67 (1969): 189-218.

13. Engelmeir RL. "Autoclave custom made metal impression trays to improve infection control". Journal of Prosthetic Dentistry 58 (1987): 121-122.

14. Sykes L., et al. "Use of custom-made conformers in the treatment of ocular defects". Journal of Prosthetic Dentistry 82 (1999): 362-365.

15. Ahmad N., et al. "Prosthetic Rehabilitation of an Ocular Defect with customized Iris: A Case Report". Malaysian Dental Journal 36.2 (2014): 1-5.
16. Barman J., et al. "Prosthetic rehabilitation of an ocular defect with custom-made ocular prosthesis: A case report". IP International Journal of Maxillofacial Imaging 6.1 (2020): 20-23.

17. Kirti Somkuwar, et al. "Ocular prosthesis: Patient rehabilitation - A Case Report". Peoples Journal of Scientific Research.

18. "Ocular prosthesis in children - clinical report". Kathmandu University Medical Journal 3.9 (2005): 81-83.

19. Pascale Scuflaire. "Tips for Fitting Eye Prostheses for the Pediatric Patient After Enucleation". US Ophthalmic Review 9.1 (2016): 49-52.

20. Vinit Shah., et al. "Custom ocular prosthesis in rehabilitation of a child operated for retinoblastoma". National Journal of Maxillofacial Surgery 6.2 (2015).

21. "Prosthetic Rehabilitation of a Pediatric Patient with an Ocular Defect".

22. Pinar Cevik., et al. "Different Techniques in fabrication of ocular prosthesis". The Journal of Craniofacial Surgery 23.6 (2012): 1779-1781.

23. Vikas B Kamble., et al. "An ocular prosthesis for Geriatric Patient- A case Report". Journal of Clinical and Diagnostic Research 7.6 (2013).

24. "Fabrication of custom ocular prosthesis using a graphical grid". Pravara Medical Review (2009): 4/1

25. "Prosthetic rehabilitation of an ocular defect: A case report". International Journal of Applied Dental Science 5.1 (2019): 188-190.

26. Lylajam S., et al. "Simplest way to fabricate custom made ocular prosthesis -A case report". International Journal of Health Sciences and Research 7.6 (2017).

\section{Volume 5 Issue 8 August 2021}

(C) All rights are reserved by Janak Poudel., et al. 